\title{
Intravenous versus Inhalational Anaesthesia and Lung Ventilation-Perfusion Matching
}

\author{
$\triangle$ \\ Authors: Philip Peyton 1,2, Harry Marsh ${ }^{1}$, Bruce Thompson 3
}

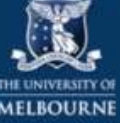

Background \& Goal of Study: The efficiency of lung gas exchange deteriorates after induction of general anaesthesia due in part to increased ventilation-perfusion (V/Q) scatter. ${ }^{1}$ Blood gas data from studies in the setting of one lung anaesthesia suggests that total IV anaesthesia (TIVA) with propofol may preserve $V / Q$ matching better than inhalational agents. ${ }^{2}$ We compared $V / Q$ matching in patients under relaxant general anaesthesia randomized to either propofol TIVA or sevoflurane maintenance anaesthesia, using both Bohr deadspace and shunt fraction measurements and the MIGET (Multiple Inert Gas Elimination Technique) for characterization of V/Q distributions. ${ }^{3}$

Materials \& Methods: Patients with clinical evidence of underlying lung disease were excluded. Baseline arterial blood sampling and collection of mixed expired gas was done before induction and repeated after 1-2 hours of relaxant general anaesthesia with controlled ventilation at an $\mathrm{FiO}_{2}$ of 0.3 , a target end-tidal $\mathrm{PCO}_{2}$ of $30-35 \mathrm{mmHg}$ and a bispectral index range of $40-60$. Blood samples for MIGET were processed by headspace equilibration in $20 \mathrm{~mL}$ gas tight glass syringes at $36^{\circ} \mathrm{C}$, with measurement of partial pressures in expired and headspace gas samples by gas chromatography. The primary endpoint was comparison of the two groups in the change from preoperative in absolute

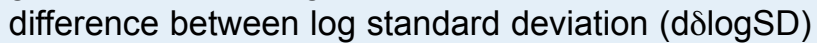
of ventilation and blood flow distributions.

Data from 20 patients (10 in each group) was complete and deemed suitable for analysis.

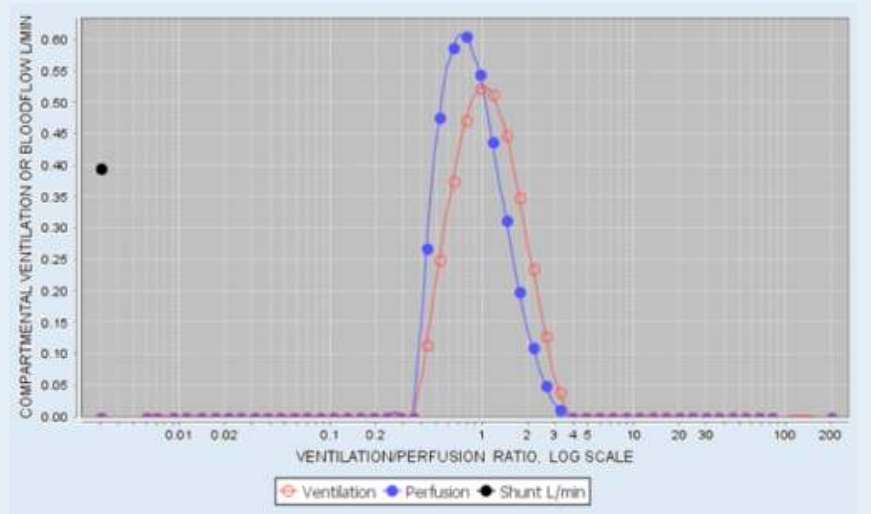

Figure 1: Pre-induction data from a patient in the sevoflurane group

$\log S \mathrm{D}$ ventilation $=0.47, \log \mathrm{SD}$ blood flow $=0.45$, $\delta \log \mathrm{SD}=0.02$
Results \& Discussion: There were no significant differences between the two groups in baseline demographics or lung function indices. Mean Bohr alveolar deadspace fraction (standard deviation SD) increased from baseline across both groups with anaesthesia [11.7 (9.1)\% to $24.7(4.8) \%, p<0.001)$ and the $\mathrm{PaO}_{2} / \mathrm{FIO}_{2}$ decreased [ 468 to $400 \mathrm{mmHg}$, log transformed $p=0.023$ ), but shunt fraction (SD) was not significantly changed $[13.1 \quad(10.0) \%$ to 10.5 (6.4) $\%, p=0.37]$.

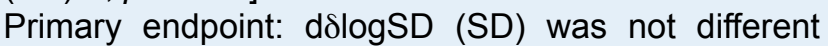
between TIVA and sevoflurane groups $(0.17(0.29)$ versus $0.17(0.85), p=0.99)$.

\begin{tabular}{|c|c|c|c|c|c|c|c|c|}
\hline \multirow{2}{*}{$\begin{array}{l}\text { Variable (unit) } \\
\text { Group }\end{array}$} & \multicolumn{2}{|c|}{ VDalv/VT (\%) } & \multicolumn{2}{|c|}{$\log \mathrm{PaO}_{2} / \mathrm{FIO}_{2}$} & \multicolumn{2}{|c|}{ Qs/Qt (\%) } & \multicolumn{2}{|c|}{ Abs diff logSD } \\
\hline & Sevo & TIVA & Sevo & TIVA & Sevo & TIVA & Sevo & TIVA \\
\hline $\begin{array}{l}\text { Preoperative } \\
\text { mean (SD) }\end{array}$ & $10.3(7.0)$ & $\begin{array}{r}12 . \\
(10 .\end{array}$ & & & & & $\begin{array}{c}0.45 \\
(0.60)\end{array}$ & $\begin{array}{c}0.13 \\
(0.14)\end{array}$ \\
\hline $\begin{array}{l}\text { Intraoperative } \\
\text { mean (SD) }\end{array}$ & $25.0(4.6)$ & $\begin{array}{l}25.0 \\
(5.2)\end{array}$ & $\begin{array}{c}2.59 \\
(0.09)\end{array}$ & $\begin{array}{l}2.59 \\
(0.13)\end{array}$ & & & $\begin{array}{l}0.62 \\
(0.64)\end{array}$ & $\begin{array}{c}0.30 \\
(0.30)\end{array}$ \\
\hline $\begin{array}{l}\text { S(difference) } \\
\text { [p value] }\end{array}$ & $\begin{array}{c}14.7 \\
k 0.001]\end{array}$ & $\begin{array}{c}12.2 \\
{[0.005]}\end{array}$ & $\begin{array}{c}0.05 \\
{[0.039]}\end{array}$ & $\begin{array}{c}0.09 \\
{[0.015]}\end{array}$ & $\begin{array}{c}-5.1 \\
{[0.233]}\end{array}$ & $\begin{array}{c}0.4 \\
{[0.879]}\end{array}$ & $\begin{array}{c}0.17 \\
{[0.515]}\end{array}$ & $\begin{array}{c}0.17 \\
{[0.097]}\end{array}$ \\
\hline$d \delta$ (Sevo vs TIVA) & \multicolumn{2}{|c|}{-2.5} & \multicolumn{2}{|c|}{0.04} & \multicolumn{2}{|c|}{5.5} & \multicolumn{2}{|c|}{0.0} \\
\hline
\end{tabular}

$\delta=$ difference between pre- and intraoperative, Sevo $=$ sevoflurane, TIVA $=$ propofol TIVA, VDalv/VT - alveolar deadspace $Q$ Q $/ Q t=$ shunt fraction, $A b s$ diff logSD = absolute difference in log standard deviation (ventilation - blood flow)

Conclusions: We found no evidence that propofol TIVA better preserved V/Q matching in patients with normal lung function undergoing controlled ventilation compared with sevoflurane.

References:

1. Lumb AB. Nunn's Applied Respiratory Physiology, 5th edition. pp 421-59

2. Praetel, et al. Anesth Analg 2004;99:1107-13.

3. Roca J, Wagner PD. Thorax 1994 49: 815-824

Acknowledgements: Thanks to Chris Stuart-Andrews, Philip Zeglinsky and Andrew Ellis for their technical assistance.

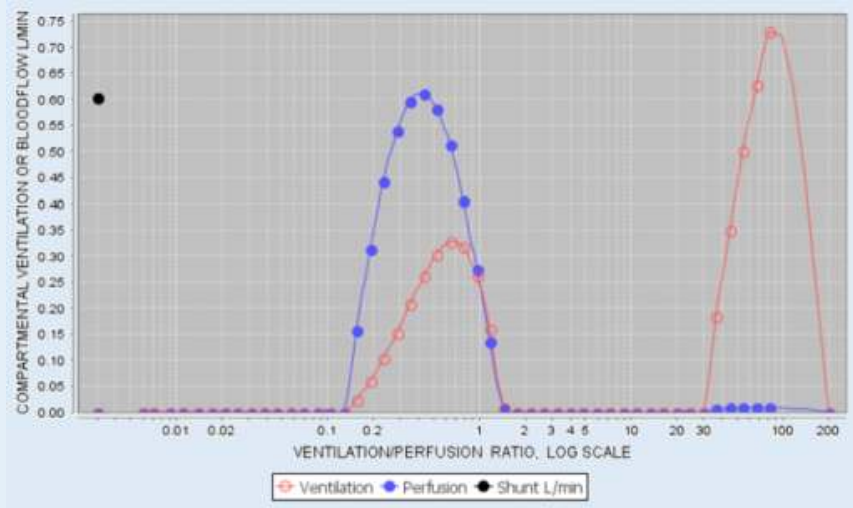

Figure 2: Post-induction data from same patient in the sevoflurane group

logSD ventilation $=2.39$, logSD blood flow $=0.69$, $\delta \log \mathrm{SD}=1.70$ 